\title{
Analysis of the literature on 3D printing in healthcare with reference to pharmaceuticals using a bibliometric approach
}

\author{
Virendra S. Ligade*(D), Akash Chimegave \\ Department of Pharmacy Management, Manipal College of Pharmaceutical Sciences, Manipal Academy of Higher Education, Manipal, India.
}

\begin{tabular}{l}
\hline ARTICLE INFO \\
\hline Received on: 27/08/2021 \\
Accepted on: 24/11/2021 \\
Available Online: 05/03/2022 \\
\\
\hline Key words: \\
3D printing, pharma, \\
pharmaceuticals, technology, \\
bibliometric, research.
\end{tabular}

\section{INTRODUCTION}

The last few decades have witnessed enormous advancements in the field of pharmaceutical drug, design, and delivery. One of the recent developments is the advent of 3DP technology (Mirza and Iqbal, 2018). The global market of pharmaceuticals has recently witnessed a new revolution in the form of 3DP technology (Chandekar et al., 2019). 3DP technology continues to push the boundaries of cost-efficiency, convenience, and customization (Banks, 2013). Approximately 30 years since its introduction, 3DP technology is set to revolutionize research and teaching laboratories. With regard to research settings, 3DP has been

\footnotetext{
*Corresponding Author

Virendra S. Ligade, Department of Pharmacy Management, Manipal College of Pharmaceutical Sciences, Manipal Academy of Higher Education, Manipal, India.E-mail:virendra.sl@manipal.edu
}

limited to biomedical applications and engineering, although it has shown tremendous potential in the chemical sciences (Gross et al., 2014). 3D printing is forecast to revolutionize drug delivery, moving away from mass manufacture toward small-batch personalized medicines. For the first time, personalized 3D-printed medicines have been evaluated in a clinical study in pediatrics to treat a rare metabolic disease (Trenfield et al., 2019). Recent approval of Spritam ${ }^{\circledR}$ and publication of United States Food and Drug Administration technical guidance on additive manufacturing related to medical devices has led to an extensive research in various fields of drug delivery systems and bioengineering (Jacob et al., 2020), identifying the key progress points that have been made on the use of 3D printing to manufacture microneedles (MNs) in the past 3 years. The study revealed that the printing resolution and the material properties are the two critical parameters that have the most influential effect on the outcome of every MN-printing endeavor (Economidou and Douroumis, 2021). $\mathrm{MN}$ technology shows excellent potential in controlled drug delivery, which has received much attention from investigators and clinics. 
Recently, three-dimensional (3D) printing has given the prototyping and manufacturing methods the flexibility to produce MN patches in a one-step manner with high levels of shape complexity and duplicability (Elahpour et al., 2021).

A large number of original articles, systematic reviews, short articles, etc. have been published in recent years on 3DP in pharmaceuticals. To date, little work has been devoted to summarizing worldwide trends in 3DP in pharmaceutical research publications. To the best of our understanding, this is the first article on the publishing patterns in 3DP in pharmaceuticals. The aim of the present study is to analyze the research publication trends in 3DP in pharmaceuticals using the bibliometric method. Bibliometrics is a statistical discipline investigating the publication patterns and trends in a certain academic field (Senel and Bas, 2020). The findings of the study can provide an overall scenario of what types of research work are more common around the world and where the focus lies.

\section{MATERIALS AND METHODS}

\section{Search tool and strategy}

We used PubMed as the search engine. PubMed was chosen because it is widely used and is recognized as the primary tool for scholars. This database is freely accessible for researchers throughout world. It covers the maximum number of quality research articles in the medical field. PubMed was chosen because it is recognized as the primary tool for scholars in the medical field (Islamaj Dogan et al., 2009; Lu, 2011; Ma et al., 2016) and it remains the optimal tool in biomedical electronic research (Falagas et al., 2008). Almost 5 million queries are issued to PubMed each day by users around the world (Islamaj Dogan et al., 2009), who rely on frequent access to be updated on biomedicine and make discoveries in their own fields (Lu, 2011). A literature search was carried out to extract all papers related to 3DP in pharmaceuticals. Keywords used for the study were "3D printing," "pharma," and "pharmaceuticals". Various publications from PubMed were collected for an 11-year period from January 1, 2010, to December 31, 2020, and then data were entered in Microsoft Office Excel sheets manually.

\section{Data analysis}

Secondary data were collected in Microsoft Office Excel sheets based on the findings from PubMed to conduct the data analysis. A total of 1,600 publications were retrieved the PubMed database. The information was processed using applications such as Microsoft Office Excel and Word. The analysis consists primarily of descriptive data that are analyzed, interpreted, and expressed in tables and percentages. Insights are categorized based on year of publication, document type, authors, publication source titles, country, research areas, etc. The keyword occurrences and country citations were visualized with help of Tableau software (http:/www.tableau.com, Tableau 2021.1), a visual analytics platform. It is a tool for exploring, analyzing, and presenting data in a visual, interactive format.

\section{RESULTS}

\section{Number of publications, study types, and research areas}

An increasing trend in research publications involving applications of 3DP in pharmaceuticals was observed. Analyses
Table 1. Year-wise distribution of publications.

\begin{tabular}{ccc}
\hline Publication year & $\begin{array}{c}\text { Number of } \\
\text { publications }\end{array}$ & \% of publication \\
\hline 2020 & 234 & 23.21 \\
2019 & 229 & 22.81 \\
2018 & 189 & 18.75 \\
2017 & 122 & 12.00 \\
2016 & 90 & 9.02 \\
2015 & 85 & 8.33 \\
2014 & 32 & 3.37 \\
2013 & 5 & 0.49 \\
2012 & 5 & 0.49 \\
2011 & 9 & 0.89 \\
2010 & 6 & 0.59 \\
\hline
\end{tabular}

Table 2. Document types in the scientific literature.

\begin{tabular}{ccc}
\hline $\begin{array}{c}\text { Document types in the } \\
\text { scientific literature }\end{array}$ & Total number & \% of publication \\
\hline Original article & 673 & 66.89 \\
Review article & 175 & 17.36 \\
Editorial & 17 & 1.68 \\
Comparative study & 16 & 1.58 \\
Evaluation study & 9 & 0.89 \\
Communication & 6 & 0.59 \\
Validation study & 3 & 0.29 \\
Technical note & 2 & 0.19 \\
Case report & 3 & 0.29 \\
Comment & 1 & 0.09 \\
Other & 101 & 10.01 \\
\hline
\end{tabular}

Table 3. Top 10 topics of research areas in the scientific literature.

\begin{tabular}{ccc}
\hline Research areas & Total number & \% of publication \\
\hline Dosage forms & 518 & 51.38 \\
Tissue engineering & 253 & 25.09 \\
Bioprinting & 136 & 13.49 \\
Implants & 39 & 3.86 \\
Wound healing & 10 & 0.99 \\
Dental & 9 & 0.89 \\
Orthology & 7 & 0.69 \\
COVID-19 & 7 & 0.69 \\
Oncology & 4 & 0.39 \\
Cardiology & 2 & 0.19 \\
\hline
\end{tabular}

showed that the number of research publications increased significantly. An upward trend in the growth of publications is witnessed for the years 2020 and 2019 with $23.21 \%$ and $22.81 \%$ of records, respectively. The least active years were 2012 and 2013 with $0.49 \%$ of records, respectively (Table 1 ). Among the studies conducted, original articles, reviews, comparative study, and editorials contributed to $66.89 \%, 17.36 \%, 1.68 \%$, and $1.58 \%$ of the records, respectively (Table 2). 
Table 4. Top 10 countries with the highest research publications.

\begin{tabular}{ccc}
\hline Top 10 countries & Total number & \% of publication \\
\hline USA & 203 & 20.13 \\
China & 153 & 15.17 \\
UK & 153 & 15.17 \\
Germany & 69 & 6.84 \\
Republic of Korea & 48 & 4.76 \\
Italy & 41 & 4.06 \\
India & 35 & 3.47 \\
Canada & 23 & 2.28 \\
Japan & 21 & 2.08 \\
Switzerland & 20 & 1.98 \\
\hline
\end{tabular}

Table 5. Top 10 journals with the highest research publications.

\begin{tabular}{ccc}
\hline Journals & Total number & \% of publication \\
\hline International Journal of Pharmaceutics & 107 & 10.61 \\
Biofabrication & 69 & 6.84 \\
Journal of Pharmaceutical Sciences & 57 & 5.65 \\
Acta Biomaterialia & 48 & 4.76 \\
Pharmaceutics & 42 & 4.16 \\
Advanced Healthcare Materials & 30 & 2.97 \\
Biomedical Materials & 27 & 2.67 \\
Materials Science and Engineering & 25 & 2.48 \\
Scientific Reports & 23 & 2.28 \\
AAPS PharmSciTech & 23 & 2.28 \\
\hline
\end{tabular}

The major topics of the research area found in the literature are presented in Table 3. It was observed that dosage form in 3DP pharmaceuticals was the key studied topic. Other key areas of focus include tissue engineering, bioprinting, implants, wound healing, dental, and orthology (Table 3). This is also evident and is reflected in the occurrence of keywords analysis (Fig. 2).

\section{Countries from which publications originated, citations, and top journal sources}

Our retrieved publications indicated that the United States was the leading country in the literature with 203 publications (20.13\%), followed by the China, United Kingdom, and Germany ( $n=153,15.17 \% ; n=153,15.17 \%$; and $n=69,6.8 \%$, respectively). Other countries with research publications were Republic of Korea, Italy, India, Canada, Japan, and Switzerland with 4.76\%, $4.06 \%, 3.47 \%, 2.28 \%, 2.08 \%$, and $1.98 \%$, respectively (Table 4 ). Name of journal sources was recorded for all manuscripts. Among the leading journals, the top contributions in said areas were in the International Journal of Pharmaceutics, Biofabrication, Journal of Pharmaceutical Sciences, Acta Biomaterialia, and Pharmaceutics with $10.61 \%, 6.84 \%, 5.65 \%, 4.76 \%$, and $4.16 \%$, respectively (Table 5).

The quality of scientific works of researchers is judged through citations received. The more citations received by individual researchers, the more favorable the growth and image of those countries in the scientific discipline. In 3DP research publication trends, researchers from USA received the highest number of citations $(n=2,105)$, followed by few leading countries, namely UK $(n=2,090)$, China $(n=1,711)$, India $(n$ $=848)$, Germany $(n=707)$, Spain $(n=656)$, Italy $(n=452)$, Republic of Korea $(n=376)$, Poland $(n=316)$, and Japan $(n$ $=302$ ). The density world map of the top countries in terms of research publication citations is shown in Figure 1.

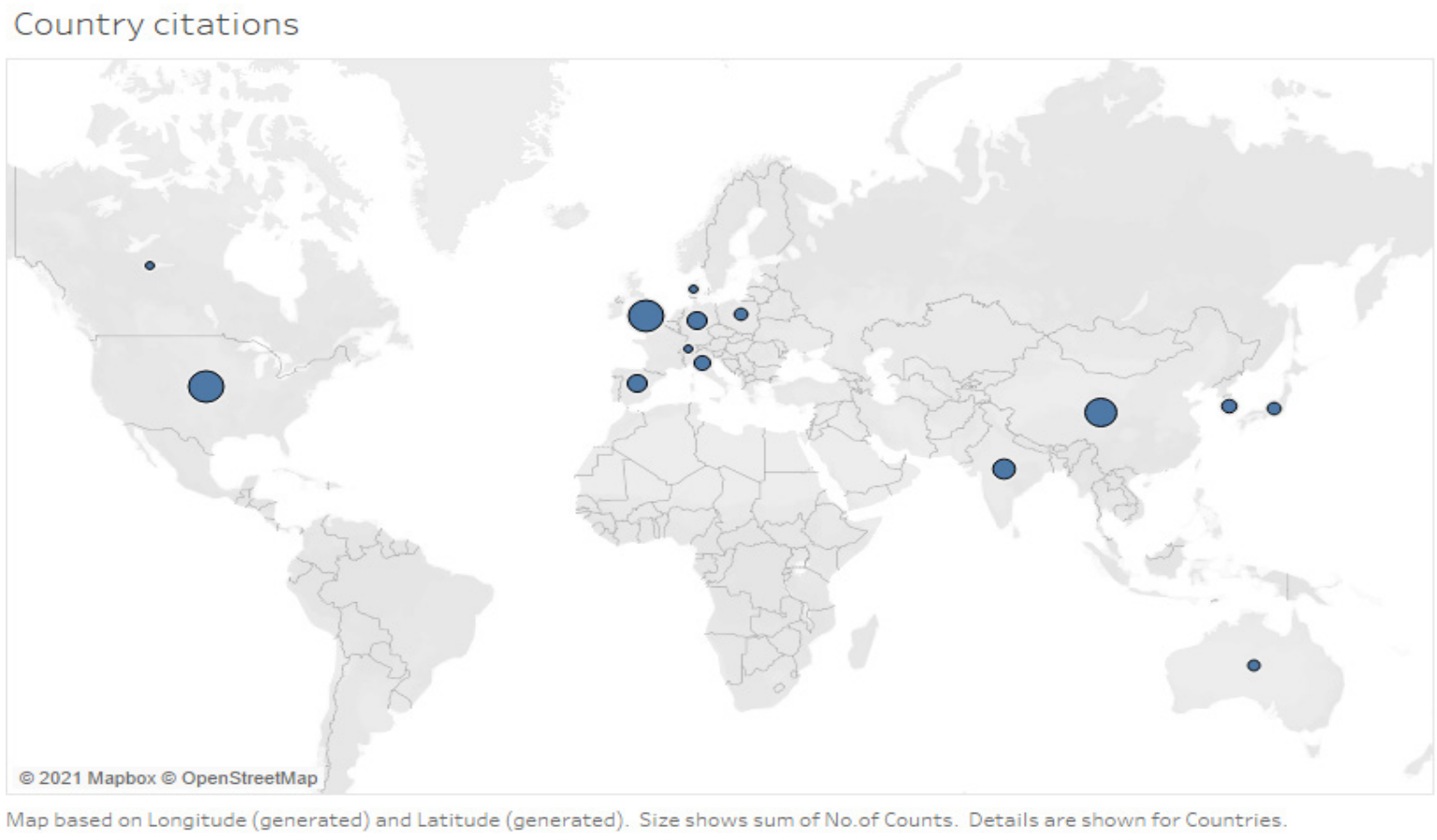

Figure 1. Density world map of the top countries in terms of research publication citations. 
Table 6. Top 10 authors with the highest research publications.

\begin{tabular}{ccc}
\hline Authors & $\begin{array}{c}\text { Total number of } \\
\text { articles }\end{array}$ & \% of publication \\
\hline Alvaro Goyanes & 42 & 4.16 \\
Sarah J. Trenfield & 14 & 1.38 \\
Niklas Sandler & 13 & 1.28 \\
Atheer Awad & 12 & 1.19 \\
Tatsuaki Tagami & 8 & 0.79 \\
Fabrizio Fina & 8 & 0.79 \\
Georgios K. Eleftheriadis & 7 & 0.69 \\
Yan Yang & 6 & 0.59 \\
Alice Melocchi & 6 & 0.59 \\
Jiaxiang Zhang & 6 & 0.59 \\
\hline
\end{tabular}

\section{Top authors and highly cited manuscripts}

Alvaro Goyanes is found to be the most productive author with $n=42(4.16 \%)$ publications, followed by Sarah J Trenfield, Niklas Sandler, Atheer Awad, Tatsuaki Tagami, Fabrizio Fina, Georgios K Eleftheriadis, Yan Yang, Alice Melocchi, and Jiaxiang Zhang with $1.38 \%, 1.28 \%, 1.19 \%, 0.79 \%, 0.79 \%, 0.69 \%, 0.59 \%$, $0.59 \%$, and $0.59 \%$ of the records, respectively (Table 6). The most cited publication in this field was entitled "A 3D bioprinting system to produce human-scale tissue constructs with structural integrity," with 369 citations published in the journal Nature Biotechnology. Another publication entitled "Medical applications for 3D printing: Current and projected uses" received 193 citations and was published in Pharmacy and Therapeutics. Publications that received more than 100 citations are presented in Table 7.

\section{Keyword frequency analysis}

Some features of the study on 3DP in pharmaceuticals could be reflected by the occurrence of keywords. Words such as "printing," "3D printing," "drug," "hydrogels," "tablets," "tissue," "bioprinting," "drug delivery," and "pharmaceutical" were found as high-frequency keywords in the present study with $45.13 \%$, $22.42 \%, 20.43 \%, 28.76 \%, 13.98 \%, 12.30 \%, 12.20 \%, 7.83 \%$, and $7.14 \%$, respectively. Table 8 presents the top 20 keywords that are related to the objective of the study. The density visualization map of the top 20 keywords is shown in Figure 2.

\section{Discussion}

Our bibliometric study on 3DP in pharmaceuticals from the year 2010 to 2020 indicates an exponential growth in the number of research publications. Every year, 3DP expands its uses in the pharmaceutical sector, saving and improving lives in ways that were previously unimaginable. Indeed, 3DP technology has been used widely in the healthcare and pharmaceutical sectors. With regard to the trends of field, most of the research publications were focused on advantages of 3DP technology, oral dosage forms, hydrogels, tablets, bioprinting, drug delivery, and pharmaceuticals. A

Table 7. Most cited research publications.

\begin{tabular}{|c|c|c|c|c|}
\hline Title of publication & Research area & year & Journal name & $\begin{array}{l}\text { Total number of } \\
\text { citations }\end{array}$ \\
\hline $\begin{array}{l}\text { A 3D bioprinting system to produce human-scale tissue } \\
\text { constructs with structural integrity }\end{array}$ & Bioprinting & 2016 & Nature Biotechnology & 369 \\
\hline $\begin{array}{l}\text { Medical applications for 3D printing: current and projected } \\
\text { uses }\end{array}$ & Dosage forms & 2014 & $\begin{array}{l}\text { Pharmacy and } \\
\text { Therapeutics }\end{array}$ & 193 \\
\hline 3D bioprinting for engineering complex tissues & Tissue engineering & 2016 & $\begin{array}{l}\text { Biotechnology } \\
\text { Advances }\end{array}$ & 185 \\
\hline $\begin{array}{l}\text { 3D bioprinting human chondrocytes with nanocellulose- } \\
\text { alginate bioink for cartilage tissue engineering applications }\end{array}$ & Bioprinting & 2015 & Biomacromoles & 167 \\
\hline $\begin{array}{l}\text { Microfluidic bioprinting of heterogeneous 3D tissue } \\
\text { constructs using low-viscosity bioink }\end{array}$ & Bioprinting & 2016 & Advanced Materials & 131 \\
\hline Omnidirectional printing of 3D microvascular networks. & Tissue engineering & 2011 & Advanced Materials & 124 \\
\hline $\begin{array}{l}\text { Bioprinting 3D microfibrous scaffolds for engineering } \\
\text { endothelialized myocardium and heart-on-a-chip }\end{array}$ & Bioprinting & 2016 & Biomaterials & 124 \\
\hline $\begin{array}{c}\text { Direct 3D printing of shear-thinning hydrogels into self- } \\
\text { healing hydrogels }\end{array}$ & Tissue engineering & 2015 & Advanced Materials & 121 \\
\hline 3D printing of scaffolds for tissue regeneration applications & Tissue engineering & 2015 & $\begin{array}{l}\text { Advanced Healthcare } \\
\text { Materials }\end{array}$ & 110 \\
\hline $\begin{array}{c}\text { Direct human cartilage repair using three-dimensional } \\
\text { bioprinting technology }\end{array}$ & Bioprinting & 2012 & Tissue engineering & 110 \\
\hline
\end{tabular}


Table 8. Top 20 keywords' frequency analysis

\begin{tabular}{cccccc}
\hline Keyword & $\begin{array}{c}\text { Co-occurrence } \\
\text { times }\end{array}$ & \% of total & Keyword & $\begin{array}{c}\text { Co-occurrence } \\
\text { times }\end{array}$ & \% of total \\
\hline Printing & 455 & 45.13 & Tissue engineering & 61 & 6.05 \\
3D Printing & 226 & 22.42 & Polymer & 54 & 5.35 \\
Drug & 206 & 20.43 & Technology & 49 & 4.86 \\
Hydrogels & 290 & 28.76 & Three-dimensional & 47 & 4.66 \\
Tablets & 141 & 13.98 & Extrusion & 46 & 4.56 \\
Tissue & 124 & 12.30 & Oral & 46 & 4.56 \\
Bioprinting & 123 & 12.20 & Human & 40 & 3.96 \\
Drug delivery & 79 & 7.83 & Dosage forms & 33 & 3.27 \\
Pharmaceutical & 72 & 7.14 & Drug release & 28 & 2.77 \\
Scaffolds & 68 & 6.74 & Drug delivery system & 28 & 2.77 \\
\hline
\end{tabular}

\section{Sheet 3}
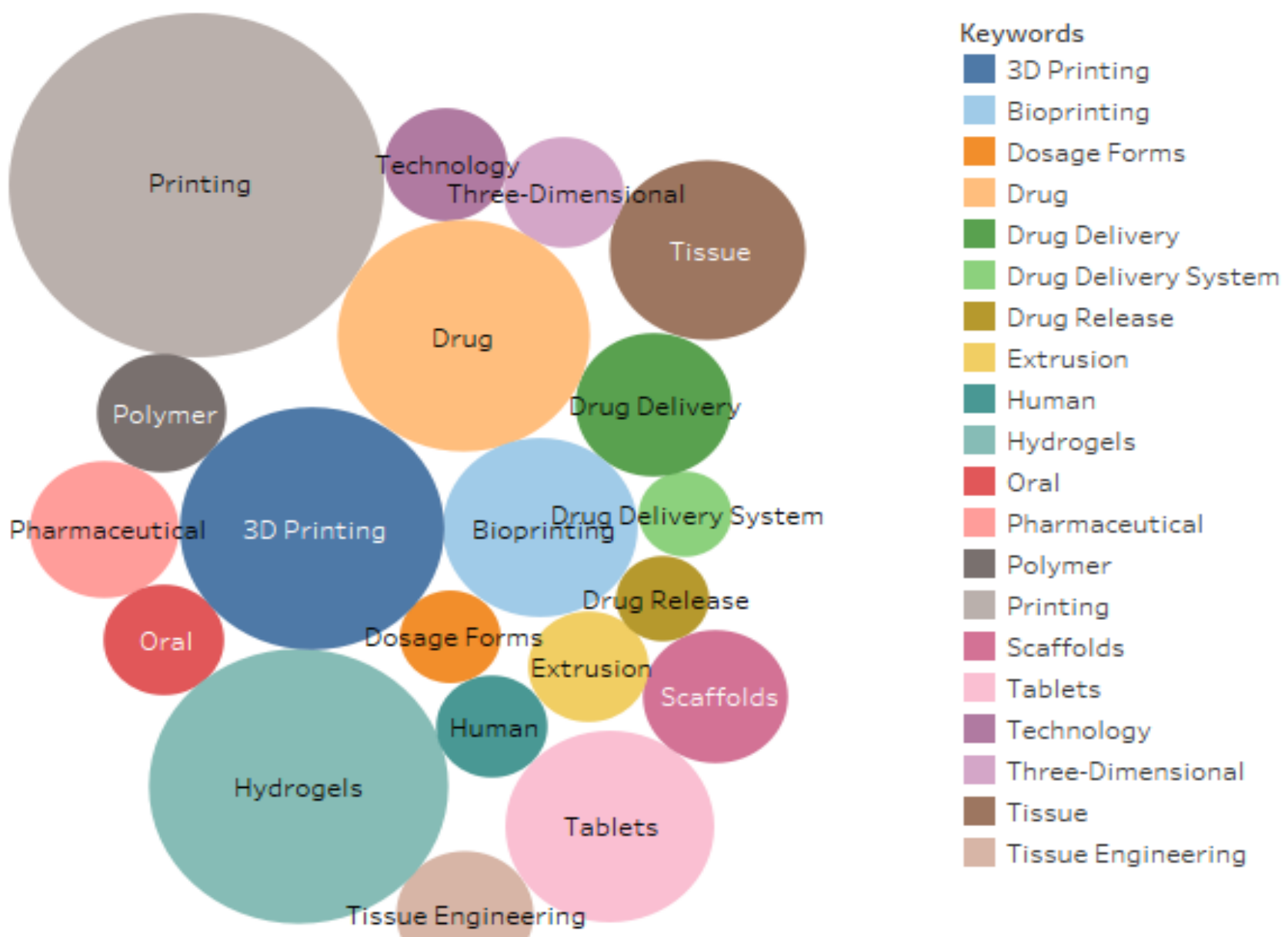

Keywords. Colour shows details about Keywords. Size shows sum of No.of Counts.

The marks are labelled by Keywords.

Figure 2. Density visualization map of the top 20 keywords. 
significant number of authors have discussed about 3DP technology, its applications, and challenges ahead. In the pharmaceutical industry, 3DP is a cutting-edge manufacturing technology and it may overcome the traditional manufacturing regime of one-size-fits-all (Azad et al., 2020). Although being in its infancy, when compared with the other sectors, year 2005 marked the rapid progression of 3DP technologies in the pharma sector (Wadher et al., 2021). This progress is also evident in terms of growth of research publications from year 2015, as shown in Table 1.

The 3DP technology is expected to transform drug delivery systems through customization of pharmaceutical formulations (Elkasabgy et al., 2020) and favorable applications in the medical sectors (Trenfield et al., 2019). Technology seems a rational approach to realize the quest for personalized drug delivery (Khan et al., 2018) and recent improvement in oral drug delivery (Pandey et al., 2020). The literature indicates the role of technology in sophisticated transdermal drug delivery systems and the range of printable materials that are presently used (Economidou et al., 2018). Various authors have highlighted the advances in technologies toward producing formulations with intended drug release (Kotta et al., 2018; Sadia et al., 2018). The application of 3DP technology for generating tablets is attracting the attention of the pharmaceutical industry (Tagami et al., 2020). Technology also favors flexible-dose drug products, polypills (polyprintlets) (Trenfield et al., 2020), and precision in dose adjustment (Karavasili et al., 2020). In addition to these advantages, extrusion-based 3DP technology is a relatively new technique (Panraksa et al., 2020). Drop-on-demand 3D bioprinting technologies currently hold the greatest potential for generating functional tissues for clinical use and for drug development (Grottkau et al., 2020). Hydrogels have been widely used as extracellular matrix materials in various 3D bioprinting applications (Krishnamoorthy et al., 2019). Authors have also deliberated on hot-melt extrusion, a technology which mixes the advantages of solid dispersion technology and mechanical preparation and is recognized in various applications in pharmaceutical formulations (Ren et al., 2019). However, issues and challenges related to 3DP are also debated in the literature. Biofabrication with various hydrogel systems allows the production of tissue or organ constructs in vitro to address various challenges in healthcare and medicine (Han et al., 2019). Technology has transformed conventional pharmaceutical operations and has focused on the design and production of dosage forms according to the patient's needs (Pereira et al., 2020).

Katakam et al. (2015) discussed about 3DP technology, its broader applications, merits, demerits, and intellectual property rights challenges. There are some regulatory challenges faced by the pharmaceutical sector and common myths surrounding the technology (Stones and Jewell, 2017). Authors in the literature have focused on many technical difficulties for 3DP of pharmaceutical products; technologies can help to overcome these challenges, in relation to marketing and lack of regulatory standards (Liang et al., 2019).

There were some limitations to our study. There was no study to compare our results within the literature. Since not all publications are indexed in PubMed, the total number of papers retrieved could be smaller than the actual number. We did a manual search and tried our best to analyze the database related to our subject, but there might have been some errors. We used only one database, PubMed, to create the rankings. However, certain authors seem to have several profiles due to the various spellings of author names or institution names.

\section{CONCLUSION}

There has been an upsurge in interests in 3DP in pharmaceuticals, as evident by an increasing trend in research publication in recent years. USA is the leading country in terms of research publications and citations. Currently, 3DP is in the primitive stage in the field of pharmaceuticals. 3DP technology has the ability to open doors in product development, manufacturing, and delivery. It has the potential to help fulfill the idea of personalized medicine, a trend that is growing rapidly in the industry. The research publication trends may continue and increase with robust studied in the field of 3DP in pharmaceuticals.

\section{AUTHOR CONTRIBUTIONS}

All authors made substantial contributions to conception and design, acquisition of data, or analysis and interpretation of data; took part in drafting the article or revising it critically for important intellectual content; agreed to submit to the current journal; gave final approval of the version to be published; and agree to be accountable for all aspects of the work. All the authors are eligible to be an author as per the international committee of medical journal editors (ICMJE) requirements/guidelines.

\section{FUNDING}

There is no funding to report.

\section{CONFLICTS OF INTEREST}

The authors report no financial or any other conflicts of interest in this work

\section{ETHICAL APPROVALS}

This study does not involve experiments on animals or human subjects.

\section{DATA AVAILABILITY}

All data generated and analyzed are included within this research article.

\section{PUBLISHER'S NOTE}

This journal remains neutral with regard to jurisdictional claims in published institutional affiliation.

\section{REFERENCES}

Azad MA, Olawuni D, Kimbell G, Badruddoza AZM, Hossain MS, Sultana T. Polymers for extrusion-based 3D printing of pharmaceuticals: a holistic materials-process perspective. Pharmaceutics, 2020; 12(2):124.

Banks J. Adding value in additive manufacturing: researchers in the United Kingdom and Europe look to 3D printing for customization. IEEE Pulse, 2013; 4(6):22-6.

Chandekar A, Mishra DK, Sharma S, Saraogi GK, Gupta U, Gupta G. 3D printing technology: a new milestone in the development of pharmaceuticals. Curr Pharm Des, 2019; 25(9):937-45.

Economidou SN, Lamprou DA, Douroumis D. 3D printing applications for transdermal drug delivery. Int $\mathrm{J}$ Pharm, 2018; 544(2):415-24

Economidou SN, Douroumis D. 3D printing as a transformative tool for microneedle systems: recent advances, manufacturing considerations and market potential. Adv Drug Deliv Rev, 2021; 173:60-9. 
Elahpour N, Pahlevanzadeh F, Kharaziha M, Bakhsheshi-Rad HR, Ramakrishna S, Berto F. 3D printed microneedles for transdermal drug delivery: a brief review of two decades. Int J Pharm, 2021; 597:120301.

Elkasabgy NA, Mahmoud AA, Maged A. 3D printing: an appealing route for customized drug delivery systems. Int J Pharm, 2020; 588:119732.

Falagas ME, Pitsouni EI, Malietzis GA, Pappas G. Comparison of PubMed, Scopus, Web of Science, and Google Scholar: strengths and weaknesses. FASEB J, 2008; 22(2):338-42.

Gross BC, Erkal JL, Lockwood SY, Chen C, Spence DM. Evaluation of 3D printing and its potential impact on biotechnology and the chemical sciences. Anal Chem, 2014; 86(7):3240-53.

Grottkau BE, Hui Z, Pang Y. A novel 3D bioprinter using directvolumetric drop-on-demand technology for fabricating micro-tissues and drug-delivery. Int J Mol Sci, 2020; 21(10):3482.

Islamaj Dogan R, Murray GC, Névéol A, Lu Z. Understanding PubMed user search behavior through log analysis. Database (Oxford), 2009; 2009: bap018.

Jacob S, Nair AB, Patel V, Shah J. 3D printing technologies: recent development and emerging applications in various drug delivery systems. AAPS Pharm Sci Tech, 2020; 21(6):220.

Han WT, Jang T, Chen S, Chong LSH, Jung HD, Song J. Improved cell viability for large-scale biofabrication with photo-cross linkable hydrogel systems through a dual-photoinitiator approach. Biomater Sci, 2019; 8(1):450-61.

Karavasili C, Gkaragkounis A, Moschakis T, Ritzoulis C, Fatouros DG. Pediatric-friendly chocolate-based dosage forms for the oral administration of both hydrophilic and lipophilic drugs fabricated with extrusion-based 3D printing. Eur J Pharm Sci, 2020; 147:105291.

Katakam P, Dey B, Assaleh FH, Hwisa NT, Adiki SK, Chandu BR, Mitra A. Top-down and bottom-up approaches in 3D printing technologies for drug delivery challenges. Crit Rev Ther Drug Carrier Syst, 2015; 32(1):61-87.

Khan FA, Narasimhan K, Swathi CSV, Mustak S, Mustafa G, Ahmad MZ, Akhter S. 3D Printing technology in customized drug delivery system: current state of the art, prospective and the challenges. Curr Pharm Des, 2018; 24(42):5049-61.

Kotta S, Nair A, Alsabeelah N. 3D printing technology in drug delivery: recent progress and application. Curr Pharm Des, 2018; 24(42):5039-48.

Krishnamoorthy S, Zhang Z, Xu C. Biofabrication of threedimensional cellular structures based on gelatin methacrylate-alginate interpenetrating network hydrogel. J Biomater Appl, 2019; 33(8):1105-17.

Liang $\mathrm{K}$, Brambilla $\mathrm{D}$, Leroux JC. Is $3 \mathrm{D}$ printing of pharmaceuticals a disruptor or enabler? Adv Mater, 2019; 31(5):e1805680.

$\mathrm{Lu}$ Z. PubMed and beyond: a survey of web tools for searching biomedical literature. Database (Oxford), 2011; 2011: baq036.

Ma Y, Dong M, Zhou K, Mita C, Liu J, Wayne PM. Publication trends in acupuncture research: a 20 -year bibliometric analysis based on PubMed. PLoS One, 2016; 11(12):e0168123.
Mirza MA, Iqbal Z. 3D printing in pharmaceuticals: regulatory perspective. Curr Pharm Des, 2018; 24(42):5081-3.

Pandey M, Choudhury H, Fern JLC, Kee ATK, Kou J, Jing JLJ, Her HC, Yong HS, Ming HC, Bhattamisra SK, Gorain B. 3D printing for oral drug delivery: a new tool to customize drug delivery. Drug Deliv Transl Res, 2020; 10(4):986-1001.

Panraksa P, Udomsom S, Rachtanapun P, Chittasupho C, Ruksiriwanich W, Jantrawut P. Hydroxypropyl methylcellulose E15: a hydrophilic polymer for fabrication of orodispersible film using syringe extrusion 3D printer. Polymers (Basel), 2020; 12(11):2666.

Pereira GG, Figueiredo S, Fernandes AI, Pinto JF. Polymer selection for hot-melt extrusion coupled to fused deposition modelling in pharmaceutics. Pharmaceutics, 2020; 12(9):795.

Ren Y, Mei L, Zhou L, Guo G. Recent perspectives in hot melt extrusion-based polymeric formulations for drug delivery: applications and innovations. AAPS Pharm Sci Tech, 2019; 20(3):92.

Sadia M, Arafat B, Ahmed W, Forbes RT, Alhnan MA. Channelled tablets: an innovative approach to accelerating drug release from 3D printed tablets. J Control Release, 2018; 269:355-63.

Senel E, Bas Y. Evolution of telepathology: a comprehensive analysis of global telepathology literature between 1986 and 2017. Turk Patoloji Derg, 2020; 36(3):218-26.

Stones JA, Jewell CM. 3D printing of pharmaceuticals: patent and regulatory challenges. Pharm Pat Anal, 2017; 6(4):145-9.

Tagami T, Ito E, Hayashi N, Sakai N, Ozeki T. Application of 3D printing technology for generating hollow-type suppository shells. Int J Pharm, 2020; 589:119825.

Trenfield SJ, Awad A, Madla CM, Hatton GB, Firth J, Goyanes A, Gaisford S, Basit AW. Shaping the future: recent advances of 3D printing in drug delivery and healthcare. Expert Opin Drug Deliv, 2019; 16(10):1081-94.

Trenfield SJ, Tan HX, Goyanes A, Wilsdon D, Rowland M, Gaisford S, Basit AW. Non-destructive dose verification of two drugs within 3D printed polyprintlets. Int J Pharm, 2020; 577:119066.

Wadher K, Trivedi R, Wankhede N, Kale M, Umekar M. 3D printing in pharmaceuticals: an emerging technology full of challenges. Ann Pharm Fr, 2021; 79(2):107-18.

\section{How to cite this article:}

Ligade VS, Chimegave A. Analysis of the literature on 3D printing in healthcare with reference to pharmaceuticals using a bibliometric approach. J Appl Pharm Sci, 2022; 12(03):096-102. 